\title{
SISO Detection over Linear Channels with Linear Complexity in the Number of Interferers
}

\author{
Giulio Colavolpe, Senior Member, IEEE, Dario Fertonani, and Amina Piemontese, Student Member, IEEE
}

\begin{abstract}
We consider detection over linear channels impaired by additive white Gaussian noise. For this general model, which describes a large variety of scenarios, novel detection algorithms are derived by applying the sum-product algorithm to a suitablydesigned factor graph. Being soft-input soft-output (SISO) in nature, the proposed detectors can be adopted in turbo processing without additional modifications. Among various applications, we focus on channels with known intersymbol interference, on frequency-division-multiplexed systems where adjacent signals are allowed to overlap in frequency to increase the spectral efficiency, and on code division multiple access systems. When compared with the existing interference-cancellation algorithms, the proposed schemes result very appealing in terms of tradeoff between performance and computational complexity. Particularly, the proposed schemes can approach or even outperform the performance provided by much more complex algorithms.
\end{abstract}

\section{INTRODUCTION}

We consider linear modulations over linear channels impaired by additive white Gaussian noise (AWGN). In a very general form, the relationship between the transmitted sequence $\boldsymbol{c}=\left[c_{1}, c_{2}, \ldots, c_{N}\right]^{\mathrm{T}}$ and the received sequence $\boldsymbol{y}=\left[y_{1}, y_{2}, \ldots, y_{K}\right]^{\mathrm{T}}$ can be written as [1]

$$
\boldsymbol{y}=\boldsymbol{H} \boldsymbol{c}+\boldsymbol{w}
$$

where $\boldsymbol{w}=\left[w_{1}, w_{2}, \ldots, w_{K}\right]^{\mathrm{T}}$ are independent and identically distributed zero-mean Gaussian random variables, while $\boldsymbol{H}$ is a matrix with $K$ rows and $N$ columns. ${ }^{1}$ We consider the general case when all terms in (1) are complex-valued, assuming that the real and imaginary components of the noise samples $w_{i}$ are independent and have the same variance $\sigma^{2}$. The model (1), which will be studied under the assumption of perfect knowledge of the matrix $\boldsymbol{H}$ and the variance $\sigma^{2}$, provides a general description for different communication schemes employing linear modulations over linear AWGN channels. For example, the matrix $\boldsymbol{H}$ can represent singleand multi-carrier transmissions over frequency-selective and possibly time-varying channels [1]-[7], multiple access systems [8]-[13], channels with multi-dimensional intersymbol interference [14], [15], or even space-time architectures [16], [17].

Submitted: January 11, 2011. Revised: June 26, 2011 and September 26 2011. Parts of this paper were presented at the IEEE Global Telecommunications Conference, New Orleans, LA, U.S.A., November-December 2008, and at the IEEE International Workshop on Signal Processing Advances for Wireless Communications, Perugia, Italy, June 2009.

The authors are with Università di Parma, Dipartimento di Ingegneria dell'Informazione, Viale G. P. Usberti, 181A, I-43124 Parma, Italy, e-mail giulio@unipr.it, piemontese@tlc.unipr.it. Giulio Colavolpe is the corresponding author.

${ }^{1}$ Sequences (matrices) will be written in lower-case (upper-case) bold fonts The symbols $(\cdot)^{\mathrm{T}},(\cdot)^{\mathrm{H}},\|\cdot\|$, and $E[\cdot]$ will denote the transpose operator the conjugate transpose operator, the 2-norm operator, and the statistical expectation operator, respectively. Given a generic matrix $\boldsymbol{M}$, we will denote by $M_{m, n}$ its entry at row $m$ and column $n$.
We are interested in maximum-a-posteriori (MAP) symbol detection [1] of the finite-order modulation symbols $c$, given the observation of $\boldsymbol{y}$. In any system modeled by (1), exact MAP detection can be carried out in a simple symbol-bysymbol processing only when the matrix $\boldsymbol{H}$ satisfies suitable orthogonality conditions [1]. On the other hand, in order to increase the bandwidth efficiency, it is often not convenient to pursue such orthogonality conditions in the system design [8], [13], [18], [19]. In this case, we say that interference arises and optimal MAP detection calls for algorithms whose complexity grows exponentially with the number of interferers [1]. In most practical scenarios, such a complexity is unmanageable and suboptimal approaches are mandatory.

The literature addressing suboptimal soft-input softoutput (SISO) detection algorithms for applications that can be led to the system model (1) is huge and an exhaustive survey is not feasible here. The reader is invited to refer to [20]-[27] and references therein. The picture is also made much more complex by the fact that most of the papers in the literature address only one of these applications at a time, concentrating on the aspects which are peculiar of a specific application and the relevant performance results (e.g., see [7], [9]-[15], [17], [28]), or describing low-complexity techniques which are not so general as to be used in all scenarios (e.g., see [29] and references therein). An exception is represented by [30], where the general model (1) is studied mainly assuming that $c$ is a Gaussian vector, hence with reference to linear minimummean-square-error (MMSE) estimation and Gaussian message passing.

Within this plethora of algorithms, most of them iterative, here we will consider as a benchmark only those with a complexity per iteration at most quadratic in the number of interferers, namely we will focus on interference cancellation (IC) algorithms originally proposed for code division multiple access (CDMA) systems and then extended to other applications. The reference benchmark is the algorithm proposed in [9], [10]—see also [11], [30], where the same algorithm is derived by applying the sum-product algorithm (SPA) to a suitable factor graph (FG) [31]. This algorithm was then applied in other scenarios or extended to other applications. As an example, in [12] the algorithm has been employed in frequency-division-multiplexed systems with intentional interference, in [17] has been extended to space-time architectures, and in [4]-[6] has been studied for turbo equalization [32] also investigating some approaches to further reduce the complexity. The algorithm is founded upon the approximation of the probability distribution of the interferers as a circularly-symmetric Gaussian probability density function (pdf) with the same mean and variance [11], [30]. The relevant complexity per iteration grows quadratically with the number of interferers (the number of users sharing 
the channel in the CDMA case) [9], which is still prohibitive in many practical scenarios. An algorithm whose complexity grows only linearly with the number of interferers is also presented in [9], but its performance is significantly worse.

The main contribution of this paper consists of the introduction of a novel FG describing the system, based on which, through the application of the SPA with proper schedules, we derive two algorithms for SISO detection. The considered FG is exact, since no approximation is required for its derivation, but contains cycles, and thus cannot lead to an algorithm for exact MAP symbol detection [31]. On the other hand, since the shortest cycles in the FG have length six, the proposed iterative algorithms ensure very good convergence, so that they can well approximate exact MAP symbol detection [31]. Interestingly, our algorithms are characterized by a complexity per iteration that grows only linearly with the number of interferers. To prove their effectiveness, we report simulation results and performance comparisons for some selected scenarios such as CDMA systems employing iterative detection/decoding, transmissions over channels affected by intersymbol interference (ISI) with turbo-equalization at the receiver [5], [32], and frequency-division multiplexing (FDM) systems with intercarrier interference (ICI) [12], [13].

The rationale for considering as a benchmark only the class of algorithms with complexity at most quadratic in the number of interferers is thus clear-our aim is to show that the proposed algorithms can approach or, in some cases, outperform more complex algorithms. And on the other hand it is very difficult to compare algorithms with different complexity and performance. It is in general expected that a larger complexity corresponds to a better performance but in this picture our algorithms occupy a prominent position since in a scenario where the large number of interferers makes unfeasible the algorithms with a quadratic complexity, we can count on simpler algorithms with a very good performance.

The remainder of this paper is organized as follows. Section II introduces a few definitions and recalls some basic concepts related to MAP symbol detection. In Section III, we present a novel FG describing the system and, by application of the SPA, we derive two SISO-detection algorithms. Section IV provides some examples of channels that can be led to the considered model, discussing for each of them the complexity of the optimal MAP symbol detector. Section V shows some performance/complexity comparisons between our algorithms and some reference benchmarks. Finally, Section VI concludes the paper.

\section{MAXimum-A-Posteriori Symbol Detection}

We consider MAP symbol detection of symbols $\boldsymbol{c}$, that requires the evaluation of the a posteriori probabilities (APPs) $P\left(c_{n} \mid \boldsymbol{y}\right)$ for all values of $n$ and $c_{n}$, given the observation of the received sequence $\boldsymbol{y}$. Any possible correlation between the transmitted symbols will be neglected in the detection stage [5], so that the probability mass function of the transmitted sequence can be factorized as

$$
P(\boldsymbol{c})=\prod_{n=1}^{N} P_{n}\left(c_{n}\right)
$$

where $P_{n}\left(c_{n}\right)$ is the a priori probability that the symbol $c_{n}$ is transmitted with index $n$.
The conditional pdf of the received sequence $y$ given the modulation symbols $c$ is

$$
\begin{aligned}
p(\boldsymbol{y} \mid \boldsymbol{c}) & =\left(2 \pi \sigma^{2}\right)^{-K} \exp \left(-\frac{\|\boldsymbol{y}-\boldsymbol{H} \boldsymbol{c}\|^{2}}{2 \sigma^{2}}\right) \\
& \propto \exp \left(-\frac{\|\boldsymbol{y}-\boldsymbol{H} \boldsymbol{c}\|^{2}}{2 \sigma^{2}}\right),
\end{aligned}
$$

the proportionality symbol $\propto$ denoting in this paper two quantities that differ by a factor independent of $c$, and thus irrelevant for the detection process [31]. If we define

$$
\begin{aligned}
\boldsymbol{x} & =\boldsymbol{H}^{\mathrm{H}} \boldsymbol{y} \\
\boldsymbol{G} & =\boldsymbol{H}^{\mathrm{H}} \boldsymbol{H},
\end{aligned}
$$

the 2-norm in (3) yields

$$
\begin{aligned}
\|\boldsymbol{y}-\boldsymbol{H} \boldsymbol{c}\|^{2} & =\boldsymbol{y}^{\mathrm{H}} \boldsymbol{y}-2 \operatorname{Re}\left\{\boldsymbol{c}^{\mathrm{H}} \boldsymbol{H}^{\mathrm{H}} \boldsymbol{y}\right\}+\boldsymbol{c}^{\mathrm{H}} \boldsymbol{H}^{\mathrm{H}} \boldsymbol{H} \boldsymbol{c} \\
& =\boldsymbol{y}^{\mathrm{H}} \boldsymbol{y}-2 \operatorname{Re}\left\{\boldsymbol{c}^{\mathrm{H}} \boldsymbol{x}\right\}+\boldsymbol{c}^{\mathrm{H}} \boldsymbol{G} \boldsymbol{c},
\end{aligned}
$$

so that (3) can we written as

$$
\begin{aligned}
p(\boldsymbol{y} \mid \boldsymbol{c}) & \propto \exp \left(-\frac{\boldsymbol{y}^{\mathrm{H}} \boldsymbol{y}-2 \operatorname{Re}\left\{\boldsymbol{c}^{\mathrm{H}} \boldsymbol{x}\right\}+\boldsymbol{c}^{\mathrm{H}} \boldsymbol{G} \boldsymbol{c}}{2 \sigma^{2}}\right) \\
& \propto \exp \left(\frac{2 \operatorname{Re}\left\{\boldsymbol{c}^{\mathrm{H}} \boldsymbol{x}\right\}-\boldsymbol{c}^{\mathrm{H}} \boldsymbol{G} \boldsymbol{c}}{2 \sigma^{2}}\right) .
\end{aligned}
$$

In other words, if the sequence $\boldsymbol{x}$ is available, the sequence $\boldsymbol{y}$ is irrelevant ( $\boldsymbol{x}$ is a sufficient statistic for MAP detection [1]). Note that this was expected, since the matrix multiplication in (4) is actually a matched filtering [1], [33], [34]. Reminiscent of the notation used for ISI channels which, as shown in the following, is a particular case of the very general model (1), we will call $\boldsymbol{y}$ and $\boldsymbol{x}$ as Forney and Ungerboeck observation models, respectively [33], [34].

The system model corresponding to (1) is

$$
\boldsymbol{x}=\boldsymbol{H}^{\mathrm{H}}(\boldsymbol{H} \boldsymbol{c}+\boldsymbol{w})=\boldsymbol{G} \boldsymbol{c}+\boldsymbol{\eta}
$$

where $\boldsymbol{\eta}=\boldsymbol{H}^{\mathrm{H}} \boldsymbol{w}$ is a sequence of circularly-symmetric Gaussian random variables, with mean zero and autocorrelation matrix $E\left[\boldsymbol{\eta} \boldsymbol{\eta}^{\mathrm{H}}\right]=2 \sigma^{2} \boldsymbol{G}$. It is useful to write the model in the scalar form

$$
x_{n}=G_{n, n} c_{n}+\sum_{m \neq n} G_{n, m} c_{m}+\eta_{n},
$$

which clarifies that $c_{n}$ and $c_{m}$ do not interfere with each other only if the coefficient $G_{n, m}=\sum_{k=1}^{K} H_{n, k}^{*} H_{k, m}$ is null. Hence, when the matrix $\boldsymbol{G}$ is diagonal, no interference arises, and MAP detection can be performed symbol-by-symbol. On the other hand, when $\boldsymbol{G}$ is not diagonal, the computation of the target APPs has a complexity that grows exponentially with the number of interferers [1], and is thus computationally infeasible in most practical scenarios.

Starting from model (1) and under the obvious assumption of zero-mean modulation symbols [1], we define the signalto-noise ratio (SNR) as

$$
\begin{aligned}
\mathrm{SNR} & =\frac{E\left[\|\boldsymbol{H} \boldsymbol{c}\|^{2}\right]}{E\left[\|\boldsymbol{w}\|^{2}\right]}=\frac{E\left[\boldsymbol{c}^{\mathrm{H}} \boldsymbol{H}^{\mathrm{H}} \boldsymbol{H} \boldsymbol{c}\right]}{2 \sigma^{2} K} \\
& =\frac{E\left[\boldsymbol{c}^{\mathrm{H}} \boldsymbol{G} \boldsymbol{c}\right]}{2 \sigma^{2} K}=\frac{\sigma_{c}^{2} \sum_{k=1}^{K} G_{k, k}}{2 \sigma^{2} K}
\end{aligned}
$$

where $\sigma_{c}^{2}=E\left\{\left|c_{m}\right|^{2}\right\}$. 


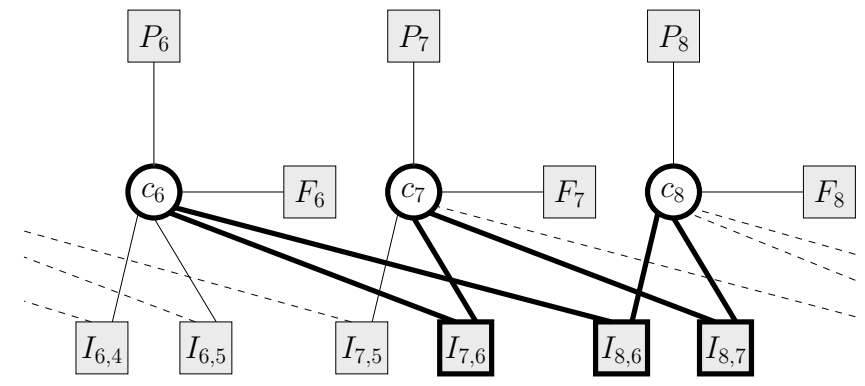

Fig. 1. Three sections of the factor graph corresponding to (12), for the case when interference between $c_{n}$ and $c_{m}$ arises only if $|m-n| \in\{1,2\}$.

\section{Proposed Graph-Based Detection Algorithms}

In this section, we introduce a novel framework for SISO detection, obtained by applying the SPA to a FG obtained from suitable manipulations of (6). Let us first write the scalar forms of the matrix operations in (6), that is

$$
\begin{aligned}
\boldsymbol{c}^{\mathrm{H}} \boldsymbol{x} & =\sum_{n=1}^{N} x_{n} c_{n}^{*} \\
\boldsymbol{c}^{\mathrm{H}} \boldsymbol{G} \boldsymbol{c} & =\sum_{n=1}^{N} G_{n, n}\left|c_{n}\right|^{2}+\sum_{n=1}^{N} \sum_{m<n} 2 \operatorname{Re}\left\{G_{n, m} c_{m} c_{n}^{*}\right\},
\end{aligned}
$$

where the Hermitian symmetry $\boldsymbol{G}=\boldsymbol{G}^{\mathrm{H}}$, obviously implied by (5), was exploited. Then, we define the functions

$$
\begin{aligned}
F_{n}\left(c_{n}\right) & =\exp \left[\frac{1}{\sigma^{2}} \operatorname{Re}\left\{x_{n} c_{n}^{*}-\frac{G_{n, n}}{2}\left|c_{n}\right|^{2}\right\}\right] \\
I_{n, m}\left(c_{n}, c_{m}\right) & =\exp \left[-\frac{1}{\sigma^{2}} \operatorname{Re}\left\{G_{n, m} c_{m} c_{n}^{*}\right\}\right],
\end{aligned}
$$

so that (6) yields

$$
p(\boldsymbol{y} \mid \boldsymbol{c}) \propto \prod_{n=1}^{N}\left[F_{n}\left(c_{n}\right) \prod_{m<n} I_{n, m}\left(c_{n}, c_{m}\right)\right] .
$$

Finally, we factorize the APP of the transmitted sequence as

$$
\begin{aligned}
P(\boldsymbol{c} \mid \boldsymbol{y}) & \propto P(\boldsymbol{c}) p(\boldsymbol{y} \mid \boldsymbol{c}) \\
& \propto \prod_{n=1}^{N}\left[P_{n}\left(c_{n}\right) F_{n}\left(c_{n}\right) \prod_{m<n} I_{n, m}\left(c_{n}, c_{m}\right)\right]
\end{aligned}
$$

by exploiting (2) and (11). Note that, when $G_{n, m}=0$, the factor $I_{n, m}$ is equal to one and can be thus dropped from the factorization (12). In practice, the node $I_{n, m}$ must be included in the FG corresponding to (12) only when $G_{n, m} \neq 0$, that is only when $c_{n}$ and $c_{m}$ interfere with each other. An example of FG is given in Fig. 1 and discussed in the relevant caption. The node $I_{n, m}$ connecting $c_{n}$ and $c_{m}$ should more rigorously be denoted as the node $I_{\max \{n, m\}, \min \{n, m\}}$, since the factor $I_{n, m}$ appears in (12) only if $n>m$. We believe that the abuse of notation is acceptable, since $I_{n, m}\left(c_{n}, c_{m}\right)=I_{m, n}\left(c_{m}, c_{n}\right)$.

We point out that the factorization (12) is exact, since no approximation was adopted in its derivation. On the other hand, the marginalization of (12) required for computing the target APPs $\left\{P\left(c_{n} \mid \boldsymbol{y}\right)\right\}$ cannot be exactly carried out by applying the SPA to the FG in Fig. 1, since it contains cycles [31], as that indicated in the figure in bold. It is easy to prove that the FG corresponding to (12) cannot contain any cycle of length lower than six, irrespectively of the number of symbols that interfere on each other. In fact, being factor nodes of at most degree 2, the necessary and sufficient condition for the arising of a cycle of length four is to have two factor nodes of degree 2 connected to the same couple of variable nodes, and this is clearly not possible, by definition of $I_{n, m}$. Hence, in our case, SPA may lead to good results since it is generally expected to provide a good approximation of the exact marginalizations when the length of the cycles is at least six (see [31] for the general treatment, and [35] for an appealing application).

The algorithm resulting from the application of the SPA to the described FG is iterative and has a complexity per iteration which is linear in the number of interferers. This is related to the adopted factorization having the appealing property that nodes $I_{n, m}\left(c_{n}, c_{m}\right)$, whose number linearly increases with the number of interferers, have degree two (i.e., they have two edges) irrespective of the number of interferers. A similar property is observed in some applications of detection over correlated flat-fading channels described in [36].

As shown in Fig. 2, we will adopt the following notation for the various messages propagated over the FG:

- $O_{n}\left(c_{n}\right)$ is the message from the variable node $c_{n}$ to the function node $P_{n}$;

- $\mu_{n, m}\left(c_{n}\right)$ is the message from the variable node $c_{n}$ to the function node $I_{n, m}$;

- $\nu_{n, m}\left(c_{n}\right)$ is the message from the function node $I_{n, m}$ to the variable node $c_{n}$.

We define $V_{n}\left(c_{n}\right)$ as the product of all messages incoming to the variable node $c_{n}$, namely

$$
V_{n}\left(c_{n}\right)=P_{n}\left(c_{n}\right) F_{n}\left(c_{n}\right) \prod_{m \neq n} \nu_{n, m}\left(c_{n}\right)
$$

The application of the SPA leads to the following rules for message updating [31]:

$$
\begin{aligned}
O_{n}\left(c_{n}\right) & =\frac{V_{n}\left(c_{n}\right)}{P_{n}\left(c_{n}\right)} \\
\mu_{n, m}\left(c_{n}\right) & =\frac{V_{n}\left(c_{n}\right)}{\nu_{n, m}\left(c_{n}\right)} \\
\nu_{n, m}\left(c_{n}\right) & =\sum_{c_{m}} I_{n, m}\left(c_{n}, c_{m}\right) \mu_{m, n}\left(c_{m}\right) .
\end{aligned}
$$

All messages $\left\{\mu_{n, m}\right\}$ and $\left\{\nu_{n, m}\right\}$ should be initialized to the same positive value - the choice of this value is irrelevant [31]. Let us discuss the various messages propagated over the graph. First, we point out the probabilistic meaning of the term $V_{n}\left(c_{n}\right)$, which is proportional to the (approximated) APP $P\left(c_{n} \mid \boldsymbol{y}\right)$, and that of the term $O_{n}\left(c_{n}\right)$, which is proportional to the (approximated) pdf $p\left(\boldsymbol{y} \mid c_{n}\right)$ and thus gives the so-called extrinsic information produced by the algorithm [31]. Then, the message $\nu_{n, m}\left(c_{n}\right)$ is proportional to the (approximated) APP of $c_{n}$, estimated locally by the node $c_{m}$ [31]. Finally, the node $I_{n, m}$ just propagates (approximated) APPs between interfering nodes, after the averaging operation (16).

Due to the presence of cycles in the considered FG, the SPA cannot lead to a unique schedule nor to a unique stopping criterion for message passing [31]. In the following, we will describe two different algorithms deriving from the adoption of two different schedules-we investigated other algorithms 


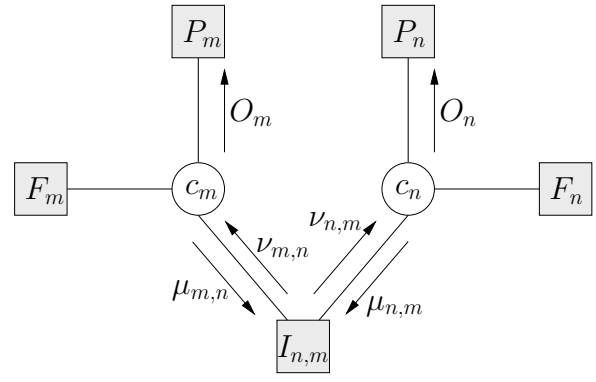

Fig. 2. Detail of the factor graph corresponding to (12) and relevant notation for the propagated messages.

that were found to provide improvements only in particular scenarios, and are thus not described here.

The first algorithm will be referred to as parallel-schedule SPA (PS-SPA). It basically exploits the fact that the lower part of the FG in Fig. 1 is formally identical to the FG describing the low-density parity-check (LDPC) codes, and adopts the same flooding schedule used for standard LDPC decoding [35]. The PS-SPA can be formalized by the following sequence of steps:

1) update all terms $\left\{V_{n}\right\}$;

2) update all messages $\left\{\mu_{n, m}\right\}$;

3) update all messages $\left\{\nu_{n, m}\right\}$;

4) if the stopping criterion is not satisfied go to step 1;

5) update all terms $\left\{V_{n}\right\}$.

We will only consider stopping criteria based on the number of self-iterations, that is on the number of times that step 4 is executed. We point out that all operations at the same step can be executed in parallel. Hence, no serial operation is required by the PS-SPA, whose latency thus does not depend on the value of $N$. This feature makes the PS-SPA very attractive for low-latency applications.

The second algorithm will be referred to as serial-schedule SPA (SS-SPA), and is inspired by the wave schedule in [37] or by shuffled LDPC decoding [38]. Let us define the forward recursion as the following sequence of steps, to be serially executed for each value of $n$ from 1 to $N$ :

a. update the messages $\left\{\nu_{n, m}\right\}_{m<n}$;

b. update the term $V_{n}$;

c. update the messages $\left\{\mu_{n, m}\right\}_{m>n}$.

Let us also define the backward recursion as the following sequence of steps, to be serially executed for each value of $n$ from $N$ down to 1 :

a. update the messages $\left\{\nu_{n, m}\right\}_{m>n}$;

b. update the term $V_{n}$;

c. update the messages $\left\{\mu_{n, m}\right\}_{m<n}$.

Finally, the SS-SPA can be formalized by the following sequence of steps:

1) run the forward recursion;

2) run the backward recursion;

3 ) if the stopping criterion is not satisfied go to step 1.

Again, we will only consider stopping criteria based on the number of self-iterations, that is on the number of times that step 3 is executed. Due to the presence of serial recursions, the SS-SPA is characterized by a latency that linearly increases with the value of $N$.
There exist some scenarios where both PS-SPA and SSSPA, when implemented in the basic version described above, provide a poor performance. After deep investigations on this behavior, we can state that the most significant problem is the overestimation of the reliability of the propagated messagesthis is a known issue of the SPA when applied to FGs with cycles [31]. A very simple way for overcoming this problem consists of adopting in (9) and (10) a value of $\sigma^{2}$ larger than the actual one. The rationale of this trick is the following: since the problem is the overconfidence in the computed messages, we can make the algorithm less confident simply by describing the channel as if it added more noise than it really does. The effectiveness of this trick, which is equivalent to weight the extrinsic information, as often made in suboptimal iterative detection/decoding schemes [32], [39], is proved by the simulation results reported in Section V.

\section{EXAMPLES OF CHANNELS}

\section{A. Channels with known ISI}

We consider a single-carrier transmission over a channel affected by known time-invariant ISI. The continuous-time equivalent channel impulse response at the receiver will be denoted by $p(t)$. Assuming ideal synchronization, at the output of a whitened matched filter the received sample at time epoch $k$ can be expressed as [2]

$$
y_{k}=\sum_{\ell=0}^{L} f_{\ell} c_{k-\ell}+w_{k} \quad, \quad k=1, \ldots, K
$$

having implicitly assumed that $c_{k}=0$ for $k<1$, and denoted by $L$ the channel memory and $\left\{f_{\ell}\right\}_{\ell=0}^{L}$ the discretetime equivalent channel impulse response. Hence, model (1) holds with $N=K$ and matrix $\boldsymbol{H}$ with entries

$$
H_{m, n}= \begin{cases}f_{m-n} & \text { for } 0 \leq m-n \leq L \\ 0 & \text { otherwise }\end{cases}
$$

Sequence $\boldsymbol{x}$ collects, in this case, the samples at the output of a filter matched to $p(t)$ (Ungerboeck observation model [34]). The optimal MAP symbol detection algorithm for this application is the so-called BCJR algorithm [40] whose complexity is linear in $K$ and exponential in $L$. This algorithm has been originally devised for the Forney model (17) and only recently extended to the Ungerboeck observation model [41].

Several algorithms have been proposed in the literature to reduce its complexity (see [42], [43] and references therein). In particular, the algorithm in [42], was derived still using the FG/SPA framework. From (2), (3), and (17) we may write [42]

$$
P(\boldsymbol{c} \mid \boldsymbol{y}) \propto \prod_{k=1}^{K} P\left(c_{k}\right) J_{k}\left(c_{k}, c_{k-1}, \ldots, c_{k-L}\right)
$$

where

$$
J_{k}\left(c_{k}, c_{k-1}, \ldots, c_{k-L}\right)=\exp \left\{-\frac{1}{2 \sigma^{2}}\left|y_{k}-\sum_{\ell=0}^{L} f_{\ell} c_{k-\ell}\right|^{2}\right\} .
$$

The corresponding FG is shown in Fig. 3 in the case of $L=2$ and $K=6$. From this figure it is clear that this factorization, derived by using the whitened matched filter output, leads to cycles of length four unless a sparse channel satisfying proper conditions is considered or proper graph transformations are 


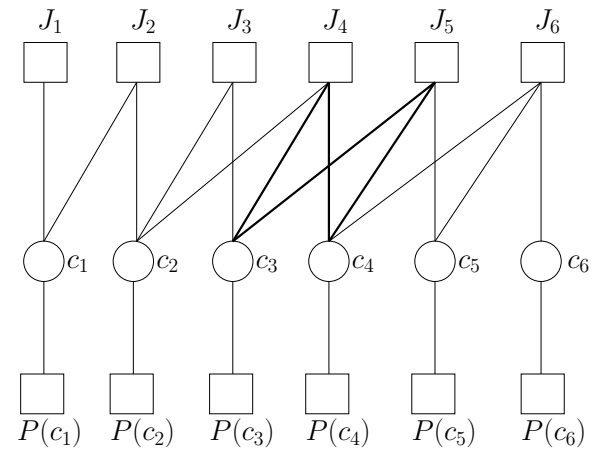

Fig. 3. Factor graph corresponding to (18).

adopted [42]. In any case, the complexity is linear in $K$ and exponential in the number of non-zero interferes [42], since this number gives the degree of nodes $J_{i}$. On the contrary, the factorization we adopted and based on the matched filter output always leads to a graph with cycles of length 6 and algorithms with a complexity per iteration which is linear in $L$.

On the other hand, the algorithm in [43] works on the trellis describing the channel memory but explores only the most promising paths, chosen according to the MAP criterion. The IC algorithm proposed in [9], [10] for CDMA systems and briefly recalled in the next section, has been also extended to the case of ISI channels in [4]-[6]. However, it is still based on the whitened matched filter output and its complexity per iteration is linear in $K$ and quadratic in $L$ when the sliding window approach described in [4]-[6] is adopted. For this reason, it will be denoted in the following as IC-Q, where "Q" stands for "quadratic". An alternative approach is described in [28], [30] based on Gaussian message passing but the complexity per iteration is still quadratic in $L$. Approximate algorithms with complexity linear in $L$ have also been derived in [4]-[6]. Among them, in the following we will consider that with the best performance and we will denote it as IC-L, where "L" stands for "linear".

The low-complexity algorithms for ISI channels in the literature (see [42], [43] and references therein) provide a satisfactory performance when the Forney observation model is adopted, but, for reasons discussed in [43], [44], do not work well when the Ungerboeck model is adopted. On the other hand, the technique proposed here works on the samples at the output of the matched filter-beside the theoretical relevance, this is of interest because the implementation of the whitening filter is critical in several practical scenarios [45], and also for applications when the detector is designed to cope only with a portion of the existing interference. In fact, in this case, a receiver working on the matched filter output results to be more robust to the unmanaged interference [46].

All these considerations straightforwardly extend to the case of a time-varying ISI channel.

\section{B. CDMA systems}

The model (1) can describe any CDMA system observed through a linear channel with AWGN [8]. In the simplest case of a synchronous CDMA system and a non-dispersive channel, the matrix $\boldsymbol{H}$ includes the spreading sequence of

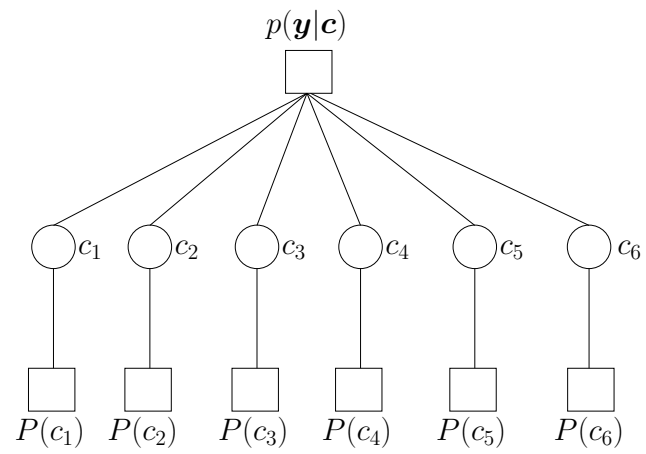

Fig. 4. Factor graph corresponding to (20).

the users, possibly scaled so that the different transmission powers (or the near-far effect) are properly accounted for [8]. In the more general case of an asynchronous CDMA system and/or a dispersive channel, the model (1) is still valid, but the expression of the matrix $\boldsymbol{H}$ is more involved [8]. For simplicity, we will here focus on a synchronous CDMA system impaired only by AWGN. In this case, the values of $N$ and $K$ represent, respectively, the number of users that share the channel and the length of the spreading sequences, measured in samples at chip rate [8], while the matched filtering (4) just consists of the correlation between the received signal and the spreading sequences of the users.

Let us now briefly recall the alternative factorizations available in the literature. From (2) we may write

$$
P(\boldsymbol{c} \mid \boldsymbol{y}) \propto p(\boldsymbol{c} \mid \boldsymbol{y}) P(\boldsymbol{c})=\exp \left\{-\frac{\|\boldsymbol{y}-\boldsymbol{H} \boldsymbol{c}\|^{2}}{2 \sigma^{2}}\right\} \prod_{k=1}^{K} P\left(c_{k}\right) .
$$

The corresponding FG is shown in Fig. 4 in the case of $N=6$. The application of the SPA to this cycle-free FG gives the optimal multiuser detector (MUD) with exponential complexity in $N$ [8]. In [9], [10] (see also [11], [30]), to reduce the complexity, when computing the marginals $P\left(c_{n} \mid \boldsymbol{y}\right)$, in the graph of Fig. 4 probabilities $P\left(c_{k}\right), k \neq n$, are replaced with complex circularly symmetric Gaussian pdfs with the same mean and variance. The resulting algorithm, still working on samples $\boldsymbol{y}$, has a complexity per iteration which is quadratic in $N$. A lower-complexity version that results if the correlation between the received samples is neglected and whose complexity is linear in $N$ has been also described in [9]-[11]. In the following, these algorithms will be referred to as IC-Q and IC-L, respectively. In this scenario, the complexity of the algorithms proposed here is linear in $N$.

\section{Spectrally-efficient FDM systems}

We consider an FDM system where $U$ synchronous and independent users simultaneously access an AWGN channel transmitting at the same power-the extension to the case of asynchronous users with different power is straightforward. We assume that all users transmit the same number of symbols $N_{u}$ and employ the same linear modulation format, whose corresponding base pulse will be denoted by $p(t)$. We also denote by $f^{(u)}$ the difference between the carrier frequency of user $u$ and the frequency assumed as reference for the computation of the complex envelope, and by $c_{n}^{(u)}$ the $M$-ary 
symbol transmitted by user $u$ at discrete-time $n$. Moreover, $c^{(u)}=\left(c_{1}^{(u)}, \ldots, c_{N_{u}}^{(u)}\right)^{\mathrm{T}}$ is the vector of the $N_{u}$ symbols transmitted by user $u$.

A set of sufficient statistics for MAP symbol detection can be obtained through a bank of $U$ filters matched to the pulses $p(t) e^{j 2 \pi f^{(u)} t}, u=1,2, \ldots, U$, and the model (7) holds with $N=U N_{u}$. The vector $c$ contains the symbols transmitted by all users, i.e., $\boldsymbol{c}=\left(\boldsymbol{c}^{(1)^{\mathrm{T}}}, \ldots, \boldsymbol{c}^{(U)^{\mathrm{T}}}\right)^{\mathrm{T}}$. The matrix $\boldsymbol{G}$ is a block matrix and we denote by $\boldsymbol{G}^{(i, j)}, i, j=1, \cdots, U$, the $(i, j)$ submatrix with $N_{u}$ rows and $N_{u}$ columns, accounting for the correlation between users $i$ and $j$. Its entries are

$$
\begin{aligned}
G_{n, m}^{(i, j)}= & e^{j 2 \pi\left(f^{(i)}-f^{(j)}\right) m T} \\
& \int p(t-(n-m) T) p^{*}(t) e^{j 2 \pi\left(f^{(i)}-f^{(j)}\right) t} d t \\
& n, m=1, \cdots, N_{u}
\end{aligned}
$$

where $T$ is the symbol period common to all users. When Nyquist pulses are employed, no ISI among symbols of the same user arises and the submatrices $G^{(i, i)}$ are diagonal.

We consider spectrally efficient FDM transmissions, where interference among adjacent users is intentionally introduced in order to increase the bandwidth efficiency [12], [13]. In other words, the spectral efficiency is increased by reducing the spacing between two adjacent channels, allowing overlap in frequency. As a consequence, the number of non-zero off-diagonal elements in the matrix $\boldsymbol{G}$ is not negligible and multiuser detection becomes necessary [12]. The optimal MUD for this scenario has a computational complexity which increases exponentially with the cardinality of the interference set. Hence, suboptimal reduced-complexity multiuser detection techniques are required. The IC-Q and IC-L algorithms, already described for CDMA systems and based on a Gaussian approximation of the multiple access interference, can be easily extended to this scenario [12], [47], [48]. Their complexity per iteration is, respectively, quadratical and linear in the cardinality of the interference set.

The proposed algorithm has a complexity per iteration which increases linearly with the cardinality of the interference set. As we will see, its performance is better than that of IC-Q and IC-L schemes for the following reason. Since we are considering an FDM scenario, even if we increase the total number of users $U$, there are in practice only a couple of adjacent users (or at most 4) that interfere with the considered user. As a consequence, the central limit theorem and the Gaussian approximation used to derive IC-Q and IC-L schemes could not be advocated.

\section{Multiple-antenna channels}

Model (1) can describe a channel with multiple transmit and receive antennas. Focusing on frequency-flat fading, $K$ and $N$ represent the number of receive and transmit antennas, respectively, whereas the entries of matrix $\boldsymbol{H}$ in (1) are the channel coefficients [16].

Computational complexity of the optimal MAP symbol detector grows exponentially with $N$. Many suboptimal solutions have been proposed in the literature (see [17] and references therein) all with complexity much larger than that of the proposed algorithm.

\section{E. OFDM systems with ICI}

In single-carrier communication systems, multipath channel spreads may cause severe ISI, which requires sophisticated and computationally-demanding equalization techniques. Transmission schemes based on orthogonal frequency-division multiplexing (OFDM) have emerged as a more convenient solution, with the key advantage that, in time-invariant channels, modulation symbols transmitted over different subcarriers do not interfere with each other even after propagating over frequency-selective channels, so that simple symbolby-symbol detection can be adopted [3]. Unfortunately, this property no longer holds on time-varying channels and/or in the presence of synchronization errors, as ICI arises [7]. In this case, the matrix $\boldsymbol{H}$ in (1) is a square matrix where the entry $H_{m, n}$ describes the ICI between the $m$-th and $n$-th subcarriers. See [49] and references therein for an extensive survey on ICI mitigation in OFDM systems and its analogy with ISI mitigation in single-carrier systems

\section{F. Multi-dimensional ISI channels}

In the previous examples, except for the spectrally-efficient FDM systems, we have considered scenarios in which interference arises in a single domain (e.g., code domain in CDMA systems, time domain in frequency-selective ISI channels, frequency domain in OFDM systems with ICI). On the other hand, the model in (1) can describe more general scenarios, in which interference among the modulation symbols arises simultaneously in different domains. For example, in the case of the spectrally-efficient FDM systems described above, interference arises in the frequency domain as well as in the time domain. See also [14] and references therein for a general treatment of bidimensional ISI, and [15] for an introduction to bidimensional ISI in storage systems.

\section{Simulation Results}

The performance of the described detection schemes is here assessed by means of computer simulations. We consider coded transmissions with iterative detection/decoding at the receiver side [5]. In such systems, the MAP symbol detector is the inner SISO detection algorithm, while, according to the turbo principle [5], the terms $\left\{P_{n}\right\}$ are iteratively updated by a SISO decoder. In the case of the IC-Q and IC-L algorithms mentioned before, the terms $\left\{P_{n}\right\}$ are used to compute soft estimates of the transmitted symbols, which are then exploited for interference mitigation [9]-[11], [30].

In the case of the proposed algorithms, extensive analyses, carried out by means of extrinsic information transfer (EXIT) charts [50] as well as bit-error rate (BER) simulations, omitted here due to the lack of space, show that it is often not convenient to execute more than one self-iteration when either the PS-SPA or the SS-SPA are employed in systems with iterative detection/decoding. Although particular scenarios were found in which self-iterations provided non negligible gains, both algorithms typically provide a satisfactory performance when, each time the SISO decoder updates the intrinsic information $\left\{P_{n}\right\}$, they execute just one self-iteration before feeding out the extrinsic information $\left\{O_{n}\right\}$. Hence, the possibility of executing more self-iterations will not be discussed hereafter.

In iterative detection/decoding schemes, a further design option consists of resetting all messages in the detection FG 


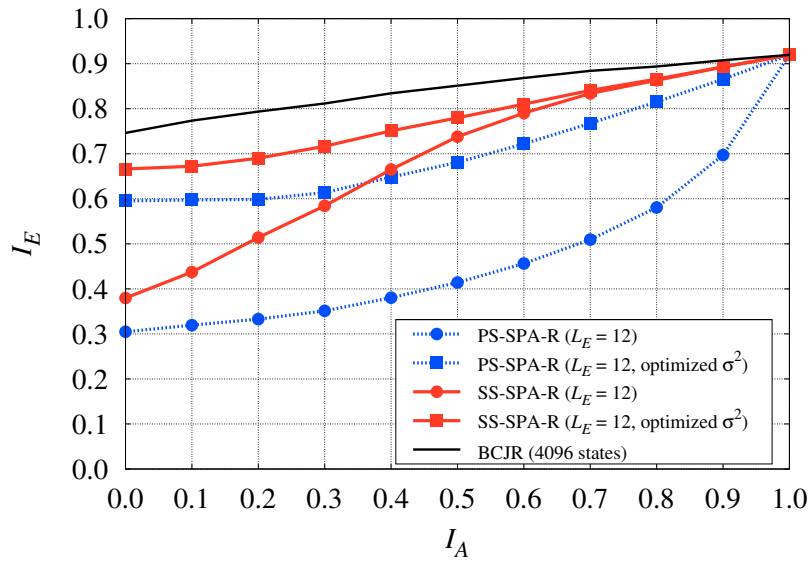

Fig. 5. EXIT charts for different algorithms over ISI channel $\mathrm{C} 1$, when a binary modulation is adopted and the value of the SNR is equal to $3 \mathrm{~dB}$.

each time the PS-SPA and the SS-SPA are provided with intrinsic information, so that any memory of the previous iterations is removed. These algorithms will be referred to as PS-SPA-R and SS-SPA-R.

\section{A. Channels with known intersymbol interference}

In this section, the performance of the proposed algorithms, implemented in the logarithmic domain [31], is assessed by means of computer simulations. All results reported in the following are related to a binary transmission with alphabet $\{ \pm 1\}$ and real-valued sequence $\left\{f_{i}\right\}$. We consider two ISI channels, referred to as $\mathrm{C} 1$ and $\mathrm{C} 2$. Channel $\mathrm{C} 1$ is characterized by a shaping pulse $p(t)=p_{\mathrm{LOR}}(t)-p_{\mathrm{LOR}}(t-T), p_{\mathrm{LOR}}(t)$ being a Lorentzian pulse with density $D=3[1]$-this model is commonly used for magnetic-storage systems. Channel C2 corresponds to faster-than-Nyquist signaling with roll-off factor $\alpha=0.1$ and time-compression factor $\tau=0.78$ [19]this system provides a very attractive spectral efficiency by introducing intentional ISI [19]. Both channels are characterized by an infinite value of $L$ and were truncated by setting $L=12,{ }^{2}$ which implies that the full-complexity BCJR works on a 4096-state trellis. To further reduce the complexity of the PS-SPA and the SS-SPA, we neglect the coefficients $\left\{G_{n, m}\right\}$ with the lowest magnitudes. Therefore, we introduce the integer parameter $L_{E} \leq L$, and define a new FG that does not include all $L$ function nodes $\left\{I_{n, m}\right\}$, but only the $L_{E}$ function nodes $\left\{I_{n, m}\right\}$ related to the coefficients $\left\{G_{n, m}\right\}$ with the largest magnitudes, so that a reduction factor equal to about $L / L_{E}$ results with respect to the original FG.

Fig. 5 reports EXIT charts related to transmissions over channel $\mathrm{C} 1$ when the value of the SNR is equal to $3 \mathrm{~dB}-$ see [50] for details on the EXIT charts related to detection algorithms, including the standard notation adopted also for labelling the axes in Fig. 5. We notice that, when the assumed value of $\sigma^{2}$ is properly optimized, the EXIT charts of the PS-SPA and the SS-SPA significantly improve with respect to when the assumed value of $\sigma^{2}$ equals the actual one-in particular, $\sigma^{2}$ is increased by $9 \mathrm{~dB}$ for the PS-SPA and by 5 $\mathrm{dB}$ for the SS-SPA. Moreover, we notice that the SS-SPA is

\footnotetext{
${ }^{2}$ We chose $L$ such that $\left|G_{m, m-i}\right| / G_{m, m}<0.01$ for $i>L$.
}

noticeably more effective than the PS-SPA in this scenario, and fairly close to the performance of the optimal BCJR.

Finally, in Fig. 6, we report the BER performance of various algorithms in a turbo equalization scheme. The results related to channel $\mathrm{C} 1$ and channel $\mathrm{C} 2$ are marked by circles and squares, respectively. An LDPC code with codeword length of 50,000 bits and rate $1 / 2$ is used in both cases. A detection instance is executed before each iteration of the SISO decoder, for a maximum of 50 iterations. The process also stops if, by checking the code syndrome, a valid codeword is found before the $50^{\text {th }}$ iteration. No interleaver is used because of the random nature of the LDPC code. In all cases, the algorithms are implemented after a (coarse) optimization of the assumed values of $\sigma^{2}$. We point out that the simulation results related to the full-complexity BCJR, yet sufficient for estimating the performance loss due to the proposed algorithms, are incomplete, since it is nearly unfeasible to obtain reliable BER curves for turbo equalizers working on a 4096-state trellis. In the case of channel $\mathrm{C} 1$, the performance of the algorithms in [4], [5] is also reported when considering only $L_{E}$ interferers for a couple of values of $L_{E}$ and employing a linear MMSE filter of length $2 L_{E}+1$ symbols.

Let us notice the impressive performance of the proposed algorithms over channel C2. First, we remark that there is no need for considering more than 5 function nodes $\left\{I_{n, m}\right\}$, since the SS-SPA practically performs as the BCJR when implemented with message resetting and $L_{E}=5$. Then, we point out that the PS-SPA loses only few tenths of $\mathrm{dB}$ when implemented without message resetting and $L_{E}=5$, and can be thus considered as the most convenient solution thanks to the low-latency properties discussed before. The proposed algorithms result just slightly less effective over channel $\mathrm{C} 1$. In particular, we notice that the SS-SPA loses about $0.7 \mathrm{~dB}$ with respect to the BCJR and is also less effective than IC-Q with $L_{E}=6$. Since, however, its complexity is much lower, as we will see in the following, this is still a very satisfactory result - to our knowledge, over this magnetic channel, the proposed solution is by far that providing the best performance/complexity tradeoff. Fig. 6 also shows the impact of the value of $L_{E}$ and message resetting in this scenario. Interestingly, the SS-SPA performs better when message resetting is implemented, while the PS-SPA does when message resetting is not implemented. The reader can also observe that the proposed SS-SPA-R algorithm with $L_{E}=3$ exhibits a very limited loss with respect to the case with $L_{E}=12$. This is due to the above mentioned property of receivers working on the matched filter output - they result to be more robust to the unmanaged interference.

Regarding the complexity of the schemes considered in Fig. 6 for channel $\mathrm{C} 1$, in Table I we report the relevant number of operations per symbol and per iteration. Note that, since the parallel/serial implementation impacts the latency but not the complexity, the table reports only one entry for both PS-SPA and SS-SPA. The reported results refer to the logarithmic-domain implementation of the algorithms, which is known to provide better numerical stability and to reduce the computational complexity [51]. The evaluation of a non linear function is counted as an access to a look-up table (LUT). In evaluating the complexity of the IC-Q algorithm, we considered the recursive implementation proposed in [9] for 
TABLE I

RECEIVERS CONSIDERED IN FIG. 6 FOR CHANNEL C1: COMPUTATIONAL LOAD PER SYMBOL AND PER ITERATION.

\begin{tabular}{|c|c|c|}
\hline Algorithm & Additions & LUT accesses \\
\hline \hline BCJR & 94199 & 16381 \\
\hline Proposed, $L_{E}=12$ & 390 & 48 \\
\hline Proposed, $L_{E}=3$ & 102 & 12 \\
\hline IC-Q, $L_{E}=6$ & 4252 & 5828 \\
\hline IC-L, $L_{E}=4$ & 100 & 120 \\
\hline
\end{tabular}

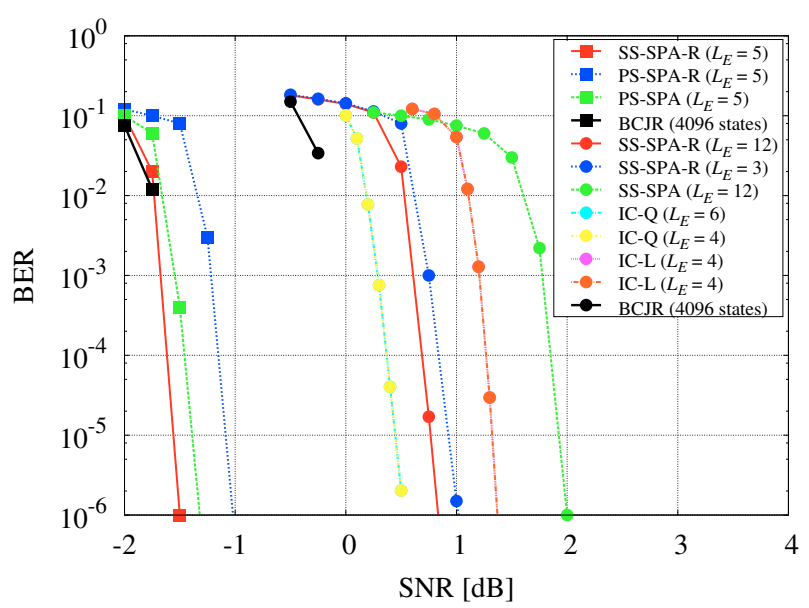

Fig. 6. Performance over ISI channels $\mathrm{C} 1$ (circles) and $\mathrm{C} 2$ (squares). A binary modulation and an LDPC code with rate $1 / 2$ are adopted.

the required matrix inversion. Even with this optimization, the matrix inversion represents the bottleneck of the algorithm from a complexity point of view. From this table it is clear the complexity advantage of the proposed algorithm. In particular, in case of channel $\mathrm{C} 1$, the proposed solution for $L_{E}=3$ has a better performance than the IC-L algorithm with $L_{E}=4$ with roughly the same complexity and it is much less complex than IC-Q algorithm with $L_{E}=6$ at the price of a limited performance loss.

Other simulation results, which are not shown here for a lack of space, confirm the effectiveness of the proposed algorithms even when non-binary modulations and different ISI channels are considered.

\section{B. CDMA systems}

In this section, we show how the proposed SISO-detection framework can be employed in CDMA systems.

First of all, we address the computational complexity of the proposed detection algorithms, assuming a binary phaseshift keying (BPSK) modulation. The number of operations per user, per symbol, and per iteration of these detection

TABLE II

CDMA SYSTEMS: COMPUTATIONAL LOAD PER USER, PER SYMBOL, PER ITERATION FOR BPSK SYMBOLS.

\begin{tabular}{|c|c|c|}
\hline Algorithm & Additions & LUT accesses \\
\hline \hline Proposed & $16 N-14$ & $2 N-2$ \\
\hline IC-Q & $2 N^{2}+8 N+1$ & $N^{2}+5 N$ \\
\hline IC-L & $9 N+2$ & $6 N+2$ \\
\hline
\end{tabular}

algorithms are reported in Table II. Note that the proposed algorithm is characterized by a complexity that increases linearly with the number of users, like the IC-L algorithm but unlike the IC-Q algorithm, whose complexity grows quadratically with $N$ [9].

Finally, we point out that the values reported in Table II were computed assuming that the $N$ users all interfere on each other, and that the detector tries to cope with all of them. In general, the value of $N$ in Table II should be read not as the number of users that actually interfere, but as the number of users that the detector assume to interfere. For example, when the number of users is large, it is mandatory for the complexity of the detector to be reduced that only a few of them are considered (clearly those characterized by the largest interference weights).

We now consider the performance. The simulation results reported in Fig. 7 refer to the case of $N=4$ users that share the channel, all transmitting with the same power. For simplicity, constant cross-correlation of the spreading sequences is also assumed, that is

$$
G_{n, m} / G_{n, n}=\gamma \in(0,1), \quad \forall m \neq n .
$$

All users employ the same non-recursive rate- $1 / 2$ convolutional code with generators $(23,35)_{8}$, a BPSK modulation, and a different random interleaver of length 256 bits. A maximum of 15 iterations between detector and decoder are allowed. We compare the BER performance of the considered algorithms versus $\gamma$, at a fixed SNR of $1.35 \mathrm{~dB}$. Aimed at low-latency detection, all algorithms are implemented in their parallel version. The performance of the optimal MAP multiuser detector is also shown for comparison. It should just be considered as a reference benchmark for the performance of the suboptimal algorithms, since its complexity is unaffordable in all practical scenarios [9].

Note that the various IC algorithms achieve the ISI-free BER (about $2.5 \cdot 10^{-5}$ ) at different values of $\gamma$, which implies that they can manage different levels of interference. Namely, the IC-Q algorithm is able to completely cancel out the interference for any value of $\gamma$ up to 0.75 , while the IC-L algorithm achieves the ISI-free performance only if $\gamma<0.1$. For the proposed PS-SPA algorithm described in Section III, in Fig. 7 we reported two curves, with and without the optimization of $\sigma^{2}$, respectively. Interestingly, the same optimization was found not to improve at all the performance of the IC-Q and IC-L algorithms. Note that the PS-SPA with optimized $\sigma^{2}$ achieves the ISI-free performance when $\gamma<0.68$. In practice, the proposed linear-complexity algorithm significantly outperforms the linear-complexity IC-L algorithm, and approaches the performance of the quadraticcomplexity IC-Q algorithm. In scenarios with a large number of users, the proposed algorithm can thus provide the most convenient performance/complexity tradeoff.

\section{Spectrally-efficient FDM systems}

Let us finally consider an FDM scenario in which $U=3$ users, equally spaced in frequency, transmit at the same power by using an octal phase-shift keying (8-PSK) modulation with a square-root raised-cosine shaping pulse having roll-off 0.35 . We define the normalized channel spacing as $F T=\left|f^{(i)}-f^{(i-1)}\right| T$. In these conditions, adjacent users 


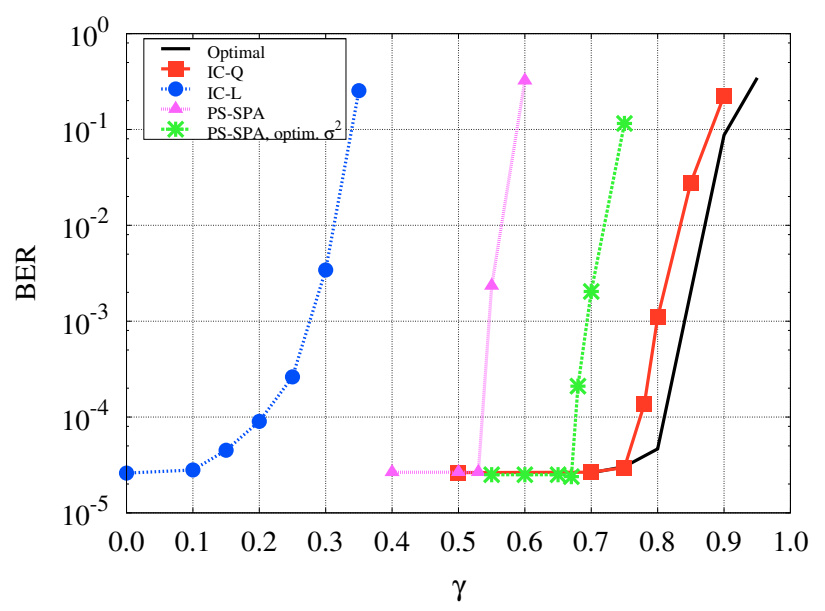

Fig. 7. CDMA system with $N=4$ synchronous users and $\mathrm{SNR}=1.35 \mathrm{~dB}$. All interferers are characterized by the same value of $\gamma$.

do not interfere when $F T=1.35$. Each user employs an outer non-recursive rate- $1 / 2$ convolutional code with generator polynomials $(23,35)_{8}$, concatenated with the Gray mapper through a random bit interleaver (different for each user). At the receiver, the described suboptimal MUDs are employed as SISO detector, and iterative detection/decoding is allowed with a maximum of 10 iterations. As a possible field of application, we consider the reverse link of a satellite system, following the Digital Video Broadcasting Return Channel via Satellite (DVB-RCS) standard [52], where a transponder is shared by multiple users using an FDM access strategy. For this reason, we consider the case of short data bursts-codewords of length 512 bits are adopted.

In Fig. 8, we show the BER curve for the middle user when using the proposed PS-SPA, the IC-Q, or the IC-L algorithm. ${ }^{3}$ Since the interference from adjacent channels dominates, we can reduce the complexity of MUD receivers assuming that the interference among non-adjacent users is negligible. In other words, $\boldsymbol{G}$ is a block tridiagonal matrix where

$$
\boldsymbol{G}^{(i, j)}=\mathbf{0} \text { for }|i-j| \geq 1 .
$$

To further limit the receiver complexity, we assume that the memory associated with the interference is of only three symbols, i.e., $G_{n, m}^{(i, i \pm 1)}=0$ for $|n-m| \geq 1$. We remark that the above approximations are exploited only by the receiver. As mentioned, all algorithms are implemented in the logarithmic domain. As far as the proposed graph-based is concerned, for computing messages $\left\{\nu_{n, m}\right\}$ as in (16) we also used the approximation $\log \left(e^{x_{1}}+e^{x_{2}}\right) \approx \max \left(x_{1}, x_{2}\right)$ [53].

In Fig. 8, we consider two channel spacings, resulting in strong-interference scenarios, and in both cases the proposed algorithm achieves the best performance among the considered suboptimal receivers. For $F T=0.55$, the PS-SPA and the IC$\mathrm{Q}$ algorithm exhibit a good performance despite the presence of strong interference, achieving the interference-free performance for $\mathrm{SNR}=8 \mathrm{~dB}$ and for $\mathrm{SNR}=9 \mathrm{~dB}$, respectively. When the spacing is $F T=0.5$, the performance gain of the PS-SPA increases. In particular, at a BER of $10^{-3}$ the proposed

\footnotetext{
${ }^{3}$ For the proposed algorithm, the detector employs the true value of $\sigma^{2}$. Hence, no optimization of the SNR assumed by the receiver was performed in this case.
}

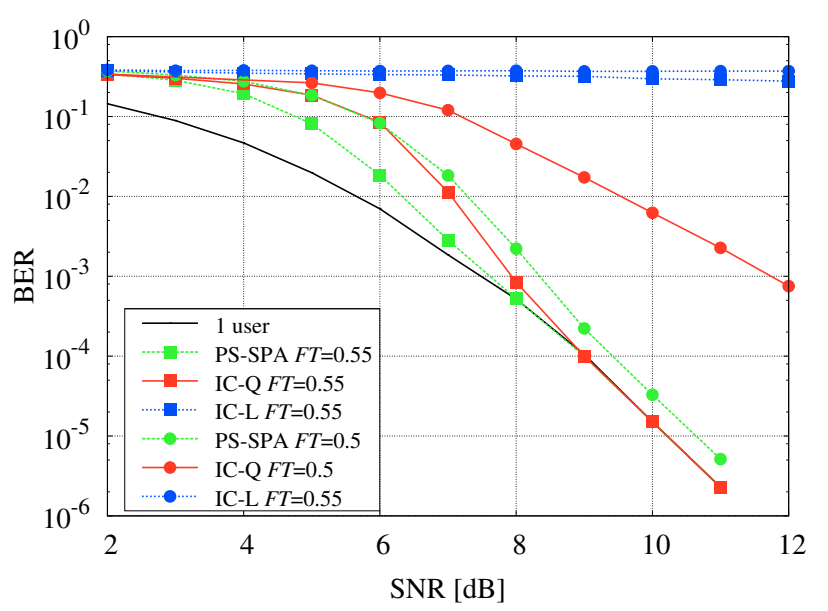

Fig. 8. FDM systems with $U=3$ synchronous users. A 8-PSK and a rate $1 / 2$ convolutional code are employed.

TABLE III

FDM SYSTEMS: COMPUTATIONAL LOAD PER SYMBOL, PER ITERATION FOR THE MIDDLE USER IN THE SCENARIO CONSIDERED IN FIG. 8.

\begin{tabular}{|c|c|c|}
\hline Algorithm & Additions & LUT accesses \\
\hline \hline Proposed & 872 & 0 \\
\hline IC-Q & 2150 & 2140 \\
\hline IC-L & 77 & 115 \\
\hline
\end{tabular}

algorithm exhibits a gain of about $3.5 \mathrm{~dB}$ with respect to the IC-Q algorithm, despite the lower computational complexity. Moreover, the loss of the proposed algorithm with respect to the interference-free case is only about $0.8 \mathrm{~dB}$. In both scenarios, the IC-L algorithm is definitely unable to manage the interference.

In Table III, we show the computational complexity of the considered MUD algorithms in the same scenario of Fig. 8. We report the number of additions between two real arguments and accesses to LUT per symbol and per iteration for the middle user in case of a logarithmic-domain implementation. The operations performed at the first iteration only have been neglected. This table clearly shows that the proposed algorithm has a complexity much lower than that of IC-Q algorithms.

Finally, we would like to give some hints on the optimization of the value of $\sigma^{2}$ assumed by the receiver. Extensive simulation results, have shown that the optimal value may strongly depend on the adopted schedule and the considered scenario (ISI channel, CDMA, or FDMA system) but is practically independent of the SNR. The dependence on the channel is often very weak-only a coarse optimization is required in all cases. As an example, in the scenario in Fig. 7 the optimal value of $\sigma^{2}$ is $5.5 \mathrm{~dB}$ larger than the real value, independently of the value of $\gamma$.

\section{CONCLUSIONS}

We have presented novel algorithms for SISO detection over linear channels impaired by additive white Gaussian noise. These algorithms, which have been derived by applying the SPA to a suitably-designed FG, are characterized by a complexity that grows only linearly with the number of interferers. 
With focus on some selected scenarios, we have shown that the proposed receivers represent a very good trade-off between performance and complexity, approaching or even outperforming the performance provided by much more complex receivers.

\section{REFERENCES}

[1] J. G. Proakis, Digital Communications. New York: McGraw-Hill, 4th ed., 2001.

[2] G. D. Forney, Jr., "Maximum-likelihood sequence estimation of digital sequences in the presence of intersymbol interference," IEEE Trans. Inform. Theory, vol. 18, pp. 284-287, May 1972.

[3] J. A. C. Bingham, "Multicarrier modulation for data transmission: An idea whose time has come," IEEE Commun. Mag., pp. 5-14, May 1990

[4] M. Tüchler, A. C. Singer, and R. Koetter, "Minimum mean square error equalization using a priori information," IEEE Trans. Signal Processing, vol. 50, pp. 673-683, Mar. 2002.

[5] M. Tüchler, R. Koetter, and A. C. Singer, "Turbo equalization: Principles and new results," IEEE Trans. Commun., vol. 55, pp. 754-767, May 2002.

[6] R. J. Drost and A. C. Singer, "Factor-graph algorithms for equalization," IEEE Trans. Signal Processing, vol. 55, pp. 2052-2065, May 2007.

[7] L. Rugini, P. Banelli, and G. Leus, "Simple equalization of time-varying channels for OFDM," IEEE Communications Letters, pp. 619-621, July 2005.

[8] S. Verdú, Multiuser Detection. Cambridge, UK: Cambridge University Press, 1998.

[9] X. Wang and H. V. Poor, "Iterative (turbo) soft interference cancellation and decoding for coded CDMA," IEEE Trans. Commun., vol. 47, pp. 1046-1061, July 1999.

[10] H. El Gamal and E. Geraniotis, "Iterative multiuser detection for coded CDMA signals in AWGN and fading channels," IEEE J. Select. Areas Commun., vol. 18, pp. 30-41, Jan. 2000.

[11] J. Boutros and G. Caire, "Iterative multiuser joint decoding: unified framework and asymptotic analysis," IEEE Trans. Inform. Theory, vol. 48, pp. 1772-1793, July 2002.

[12] B. F. Beidas, H. El Gamal, and S. Kay, "Iterative interference cancellation for high spectral efficiency satellite communications," IEEE Trans. Commun., vol. 50, pp. 31-36, Jan. 2002.

[13] A. Barbieri, D. Fertonani, and G. Colavolpe, "Time-frequency packing for linear modulations: spectral efficiency and practical detection schemes," IEEE Trans. Commun., vol. 57, pp. 2951-2959, Oct. 2009.

[14] Y. Zhu, T. Cheng, K. Sivakumar, and B. J. Belzer, "Markov random field detection on two-dimensional intersymbol interference channels," IEEE Trans. Signal Processing, pp. 2639-2648, July 2008.

[15] Y. Wu, J. A. O'Sullivan, N. Singla, and R. S. Indeck, "Iterative detection and decoding for separable two-dimensional intersymbol interference," IEEE Trans. on Magnetics, pp. 2115-2120, July 2003

[16] V. Tarokh, N. Seshadri, and A. R. Calderbank, "Space-time codes for high data rate wireless communication: Performance criterion and code construction," IEEE Trans. Inform. Theory, vol. 44, pp. 744-765, Mar. 1998.

[17] J. Hu and T. M. Duman, "Graph-based detection algorithms for layered space-time architectures," IEEE J. Select. Areas Commun., vol. 26, pp. 269-280, Feb. 2008

[18] J. E. Mazo, "Faster-than-Nyquist signaling," Bell System Tech. J., vol. 54 pp. 1450-1462, Oct. 1975.

[19] F. Rusek and J. B. Anderson, "On information rates of faster than Nyquist signaling," in Proc. IEEE Global Telecommun. Conf., (San Francisco, CA, U.S.A.), Nov. 2006.

[20] Z. Wu, Coding and iterative detection for magnetic recording channels. Kluwer Academic Publishers, 2000.

[21] K. M. Chugg, A. Anastasopoulos, and X. Chen, Iterative Detection: Adaptivity, Complexity Reduction, and Applications. Kluwer Academic Publishers, 2001.

[22] G. Ferrari, G. Colavolpe, and R. Raheli, Detection Algorithms for Wireless Communications. John Wiley \& Sons, 2004.

[23] H. Wymeersch, Iterative Receiver Design. New York, U.S.A.: Cambridge University Press, 2007

[24] "Special issue on turbo techniques: Algorithms \& applications," Proc. IEEE, vol. 95, June 2007.

[25] "Equalization techniques for wireless communications: Theory \& applications," IEEE J. Select. Areas Commun., vol. 26, Feb. 2008.

[26] M. L. Honig (ed.), Advances in Multiuser Detection. John Wiley \& Sons, 2009.
[27] L. Hanzo, B. L. Liew, B. L. Yeap, R. Y. S. Tee, and S. X. Ng, Turbo Coding, Turbo Equalisation and Space-Time Coding: EXIT-Chart-Aided Near-Capacity Designs for Wireless Channels. John Wiley \& Sons, 2011.

[28] Q. Guo and L. Ping, "LMMSE turbo equalization based on factor graph," IEEE J. Select. Areas Commun., vol. 26, pp. 311-319, Feb. 2008.

[29] D. Fertonani, A. Barbieri, and G. Colavolpe, "Novel graph-based algorithms for soft-output detection over dispersive channels," in Proc. IEEE Global Telecommun. Conf., (New Orleans, LA, USA), Nov.-Dec. 2008

[30] H.-A. Loeliger, J. Dauwels, J. Hu, S. Korl, L. Ping, and F. R. Kschischang, "The factor graph approach to model-based signal processing," Proc. IEEE, vol. 95, pp. 1295-1322, June 2007.

[31] F. R. Kschischang, B. J. Frey, and H.-A. Loeliger, "Factor graphs and the sum-product algorithm," IEEE Trans. Inform. Theory, vol. 47, pp. 498519, Feb. 2001.

[32] C. Douillard, M. Jezequel, C. Berrou, A. Picart, P. Didier, and A. Glavieux, "Iterative correction of intersymbol interference: turboequalization," European Trans. Telecommun., vol. 6, pp. 507-511, September/October 1995.

[33] G. D. Forney, Jr., "Lower bounds on error probability in the presence of large intersymbol interference," IEEE Trans. Commun., vol. 20, pp. 7677, Feb. 1972.

[34] G. Ungerboeck, "Adaptive maximum likelihood receiver for carriermodulated data-transmission systems," IEEE Trans. Commun., vol. com22, pp. 624-636, May 1974.

[35] T. Richardson and R. Urbanke, "The capacity of low density parity check codes under message passing decoding," IEEE Trans. Inform. Theory, vol. 47, pp. 599-618, Feb. 2001.

[36] G. Colavolpe, "On LDPC codes over channels with memory," IEEE Trans. Wireless Commun., vol. 5, pp. 1757-1766, July 2006.

[37] G. Colavolpe, "Design and performance of turbo Gallager codes," IEEE Trans. Commun., vol. 52, pp. 1901-1908, Nov. 2004.

[38] J. Zhang and M. P. C. Fossorier, "Shuffled iterative decoding," IEEE Trans. Commun., vol. 2, pp. 209-213, Feb. 2005.

[39] G. Colavolpe, G. Ferrari, and R. Raheli, "Extrinsic information in iterative decoding: a unified view," IEEE Trans. Commun., vol. 49, pp. 2088-2094, Dec. 2001.

[40] L. R. Bahl, J. Cocke, F. Jelinek, and J. Raviv, "Optimal decoding of linear codes for minimizing symbol error rate," IEEE Trans. Inform. Theory, vol. 20, pp. 284-287, Mar. 1974.

[41] G. Colavolpe and A. Barbieri, "On MAP symbol detection for ISI channels using the Ungerboeck observation model," IEEE Commun. Letters, vol. 9, pp. 720-722, Aug. 2005.

[42] G. Colavolpe and G. Germi, "On the application of factor graphs and the sum-product algorithm to ISI channels," IEEE Trans. Commun., vol. 53 , pp. 818-825, May 2005.

[43] D. Fertonani, A. Barbieri, and G. Colavolpe, "Reduced-complexity BCJR algorithm for turbo equalization," IEEE Trans. Commun., vol. 55, pp. 2279-2287, Dec. 2007.

[44] F. Rusek, M. Loncar, and A. Prlja, "A comparison of Ungerboeck and Forney models for reduced-complexity ISI equalization," in Proc. IEEE Global Telecommun. Conf., (Washington, DC, U.S.A.), Nov. 2007.

[45] A. Hafeez and W. E. Stark, "Decision feedback sequence estimation for unwhitened ISI channels with applications to multiuser detection," IEEE J. Select. Areas Commun., vol. 16, pp. 1785-1795, Dec. 1998.

[46] A. Modenini, G. Colavolpe, and N. Alagha, "On the achievable spectral efficiency of linear modulations with time-frequency packing." submitted to IEEE Global Telecommun. Conf. (GLOBECOM'11), 2011.

[47] D. Yachil, J. Davidson, and B. Bobrosky, "Low complexity multichannel synchronization for satellite systems with adjacent channel interference," International Journal of Satellite Communications and Networking, vol. 24, pp. 1-22, 2006.

[48] J. Grotz, B. Ottersten, and J. Krause, "Joint channel synchronization under interference limited conditions," IEEE Trans. Wireless Commun. vol. 6, pp. 3781-3789, Oct. 2007.

[49] K. Tu, D. Fertonani, T. M. Duman, M. Stojanovic, J. G. Proakis, and P. Hursky, "Mitigation of intercarrier interference for OFDM over time-varying underwater acoustic channels," IEEE Journal of Oceanic Engineering, to appear in 2011.

[50] S. ten Brink, G. Kramer, and A. Ashikhmin, "Design of low-density parity-check codes for modulation and detection," IEEE Trans. Commun., vol. 52, pp. 670-678, Apr. 2004

[51] P. Robertson, E. Villebrun, and P. Hoeher, "A comparison of optimal and suboptimal MAP decoding algorithms operating in the log domain," in Proc. IEEE Intern. Conf. Commun., (Seattle, WA), pp. 1009-1013, 1995.

[52] ETSI, "EN 301 790: DVB-RCS V1.4.1, Digital Video Broadcasting (DVB); Interaction Channel for Satellite Distribution Systems," 2003.

[53] P. Roberston, E. Villebrun, and P. Hoeher, "Optimal and sub-optimal maximum a posteriori algorithms suitable for turbo decoding," European Trans. Telecommun., vol. 8, pp. 119-125, March/April 1997. 


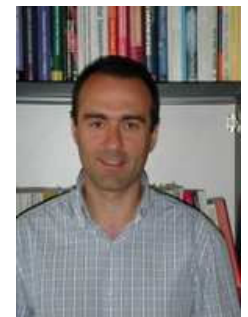

Giulio Colavolpe (S'96-M'00-SM'11) was born in Cosenza, Italy, in 1969. He received the Dr. Ing. degree in Telecommunications Engineering (cum laude) from the University of Pisa, in 1994 and the Ph.D. degree in Information Technologies from the University of Parma, Italy, in 1998. Since 1997, he has been at the University of Parma, Italy, where he is now an Associate Professor of Telecommunications at the Dipartimento di Ingegneria dell'Informazione (DII). In 2000, he was Visiting Scientist at the Institut Eurécom, Valbonne, France. His research interests include the design of digital communication systems, adaptive signal processing (with particular emphasis on iterative detection techniques for channels with memory), channel coding and information theory. His research activity has led to more than 150 papers in refereed journals and in leading international conferences, and 15 industrial patents.

He received the best paper award at the 13th International Conference on Software, Telecommunications and Computer Networks (SoftCOM'05), Split, Croatia, September 2005, the best paper award for Optical Networks and Systems at the IEEE International Conference on Communcations (ICC 2008), Beijing, China, May 2008, and the best paper award at the 5th Advanced Satellite Mobile Systems Conference and 11th International Workshop on Signal Processing for Space Communications (ASMS\&SPSC 2010), Cagliari, Italy.

$\mathrm{He}$ is currently serving as an Editor for IEEE Transactions on Wireless Communications and IEEE Wireless Communications Letters and as an Executive Editor for Transactions on Emerging Telecommunications Technologies (ETT).

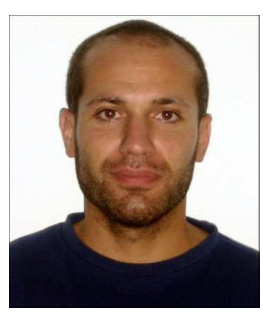

Dario Fertonani was born in Mantua, Italy, in 1980. He received the M.S. degree in telecommunications engineering in 2004 and the Ph.D. degree in information technology in 2008, both from the University of Parma, Parma, Italy. He held a postdoctorate position at Arizona State University, Tempe, AZ, from May 2008 to December 2009. Currently, he works at Qualcomm Inc., San Diego, CA.

His research interests include various topics in digital communications and information theory, with a particular focus on detection and coding for channels with memory. He is the coauthor of several publications in international journals and conferences.

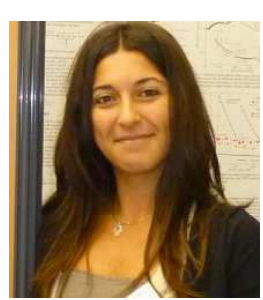

Amina Piemontese (S'09) was born in San Giovanni Rotondo, Italy, in 1980. She received the Dr.Ing. degree in Telecommunications Engineering from the University of Parma, Parma, Italy, in 2006 and the Ph.D. degree in Information Technology from the University of Parma and from TELECOM Bretagne, Brest, France, in 2011. Currently, she holds a postdoctorate position at the University of Parma. Her research activity includes various topics in digital communications, with particular emphasis on iterative joint detection and decoding algorithms and multiuser communications theory. 